\title{
Numerical analysis of the process of pipe connection with a clamping ring using the finite element method
}

\author{
Kang-Yul Bae ${ }^{\# 1}$, Young-Soo Yang ${ }^{\# 2}$ and Sungun Go ${ }^{\# 3}$ \\ ${ }^{\# 1}$ Department of Mechatronics Engineering, \\ Gyeongnam National University of Science and Technology, South Korea, \\ e-mail: kybae@gntech.ac.kr

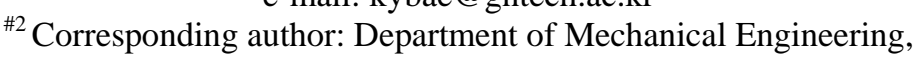 \\ Chonnam National University,77 Yongbong-ro, Bukgu, Gwangju 500-757, South Korea, \\ e-mail: ysyang@jnu.ac.kr \\ \#3 Global Production Technology Center, Samsung Electronics, South Korea
}

\begin{abstract}
The mechanical fit-up process of a pipe assembly with a clamping ring has been widely used to connect small pipes in the refrigerator and air-conditioner industries. However, few studies have been carried out to obtain the relation between the design parameters and tightening force in a pipe assembly. In this study, considering the design parameters for a pipe assembly, we modeled the mechanical process of joining the pipes with the ring, and analyzed the process using the finite element method (FEM). We then analyzed the effect of design parameters on both the distribution of radial stresses and the contact force at the contact areas, which was closely related to the tightening force of the joint. After a series of analyses to obtain the contact forces with the change of size of pipes and clamping ring, we derived a simplified equation that related the design parameters to the contact forces by the statistical technique of a recursion method. We were able to easily predict the contact forces using the simplified equation for the given sizes of pipes and clamping ring. In addition, the equation could be used to select optimal design parameters for generating the maximum contact force in a pipe assembly. Finally, we estimated the tightening force of the pipe joint from the contact forces, combined with the friction coefficient, which was determined by matching the estimated tightening force with the result of a corresponding actual tensile test.
\end{abstract}

Keyword: Pipe connection, Clamping ring, Tightening force, Optimal design, Numerical analysis

\section{INTRODUCTION}

Assembly between small pipes for refrigerators and air conditioners can be produced by the brazing process with filler material and flame heating. However, the process may deform the pipes, and also change the material properties, due to the heating procedure. Therefore, a mechanical fitting process has been developed to join two pipes together with a clamp ring [1 2]. In the joining process, an inner pipe is inserted first into an outer pipe that has a slightly greater inner diameter than the outer diameter of the inner pipe. The clamping ring then contracts the outer pipe to close contact with the inner pipe for the pipe joint to develop tightening force. Because of the effectiveness of the process, it is prevalent in the permanent assembly of small pipes in the refrigerator and air-conditioner industries [3 4]. In addition, the connection provides extraordinary sealing integrity in both liquid and gas services, and unlike other mechanically attached fittings, there are no elastomeric or rubber seals, O-rings or gaskets that can degrade and leak over time [5]. Various requirements of the related code to be improved have been studied for the application of pipe products joined by the mechanical fitting process with respect to quantifying the performance of the joint [6].

Meanwhile, the size of a clamping ring for a pipe joint in the process has usually been decided by empirical data. Therefore, it requires many experiments with a variety of diameters and materials of small pipes to select a proper clamping ring. However, even though this procedure consumes considerable cost and time, it is unable to obtain optimal design parameters. An analytical or numerical approach is needed to analyze the process for acquiring the effect of design parameters on the performance of the joint in an effective manner. Such analysis could effectively obtain optimal design parameters to provide maximum contact force for a joint for a variety of pipes.

A few numerical studies on mechanical connection processes of pipes have been reported. Hatton et al. [7] used a design approach and analysis program to study the end fitting of pipes in a deep water dynamic riser, to demonstrate reliable application in a critical and structurally demanding application. Chen et al. [8] used FEM analysis to simulate the sealing performance of a saddle-shaped sealing ring for a steel pipe assembly. Kim et al. [9] studied the variation of plastic deformation of pipes made of aluminum in a mechanical pipe fitting process with changing the shape of a fitting link; the study analyzed the effect of the thickness and leading angle of the 
link on the deformation. Lee et al. [10] analyzed the effect of the distance between the body and nut on the deformation of pipes for a mechanical fitting device proposal to join pipes with a fitting assembly that consisted of body, nut, link, and clip ring. In the drawing process of a composite material and a stainless steel pipeat the same time, Kim et al. [11] analyzed the effect of process parameters such as die angle, friction condition and drawing speed to obtain an optimal process condition to reduce residual stresses of the product. However, few studies have analyzed the contact and tightening forces of a pipe joint, which would mainly depend on the quality of the joint produced by a mechanical fitting process. Furthermore, there has been little research on the analysis of the process employed for the connection of small pipes with a clamping ring.

In this study, we proposed a numerical model based on the finite element method (FEM) for the pipe joining process with a clamping ring to analyze the contact force between the pipes. In the analysis, we obtained stress distributions in the radial direction of the model of the pipes and the clamping ring assembly to decide the contact forces at the contact surface between the two pipes. We also studied the deformed shapes of the pipes in the process to examine the change of the contact area according to the ring size. We followed a series of analyses to obtain the contact forces between the steel pipes for a total of 27 combinations of pipe sizes. After the numerical analyses, we used the statistical technique of a recursion method [12] on the obtained results and input design parameters, to derive a simplified equation that presented the relation between contact force and each design parameter of the joint. We could use the simplified equation to estimate the maximum contact force for the given design parameters, which consisted of the sizes of the pipes and the clamping ring involved.

With a numerical simulation of a tensile test on the pipe joint, we deduced the tightening force in the axial direction of the pipe joint with a clamping ring from the contact forces by considering appropriate friction coefficients between the pipes contracted by the ring. We performed a series of tensile analyses to obtain a friction coefficient that made the tightening force estimated by the numerical analysis accord with the experimental result.

\section{NUMERICAL MODEL AND ANALYSIS OF THE PIPE CONNECTION}

In a pipe joint with a clamping ring for two pipes, one the inner pipe and the other the outer pipe, which Fig. 1 shows has a slightly greater inner diameter than the outer diameter of the inner pipe, the inner pipe is first inserted into the outer pipe. Before insertion, anaerobic sealant is applied on the surface of the end of the inner pipe. The sealant flows within the space and hardens later after assembly to enclose the joint by sealing surface defects, compensating for any unevenness in the tube surfaces, such as longitudinal grooves or surface porosities [13]. Then the clamp ring, which has an inner diameter that is less than the outer diameter of the outer pipe, and has a taper at the leading section to facilitate advance onto the outer pipe, is compelled from its initial position outside the inner pipe by a hydraulic machine onto the overlapped area between the two pipes to contract the outer pipe onto the inner pipe. At the same time, this produces a radial expansion of the clamping ring in the elastic range. Finally, the tightening force of the joint can be obtained from the contact force between the two pipes by contraction for the two pipes to develop joint strength.

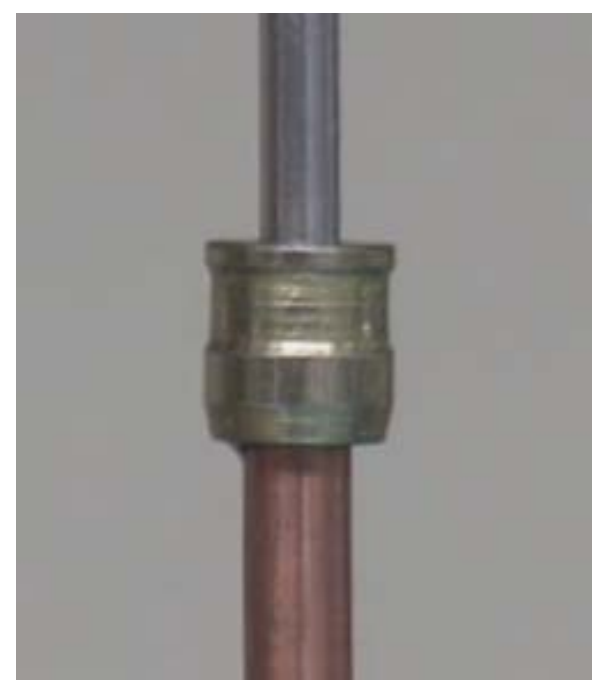

(a) Configuration of pipe connection

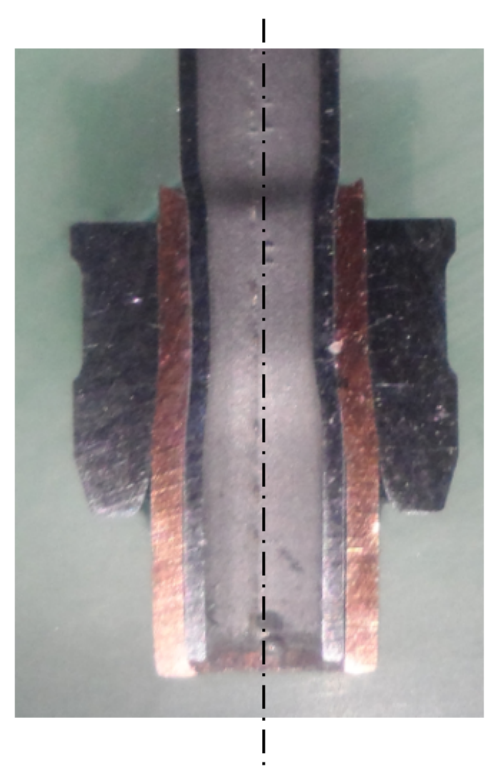

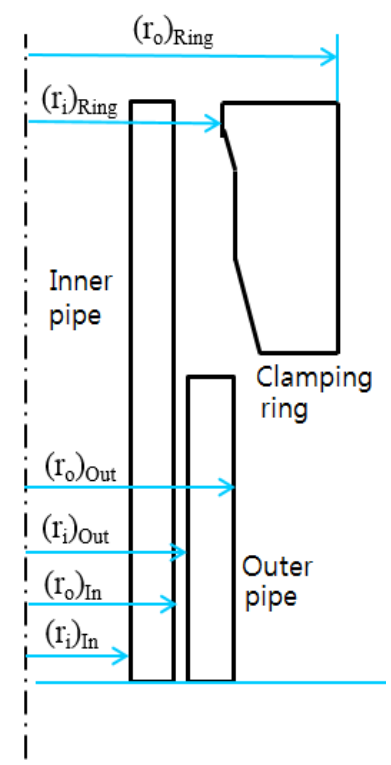

Fig. 1.Configuration of the pipe connection with a clamping ring and major design parameters.

\section{A. Numerical model of the pipe joining process}


Figure 1 shows the inner shape of a typical clamping ring that has a taper at the leading sectionslanted conically in the radial direction, and a projection in the radial direction at the trailing section. We set the overlapped length between the two pipes to $15 \mathrm{~mm}$, which is the contact length of the joint. Table 1 shows the pipes and rings used for the analyses were made of steel and their mechanical properties [14].

We modeled and analyzed the joining process with the typical ring as follows. We prepared a finite element model of the pipes and the clamping ring, including the interfaces between the pipes and the ring. We divided the contact regions into smaller areas to be refined and free-meshed so that the contact surfaces had the same mesh pattern. The model contained three contact regions to simulate the friction and manage local contact stresses.

We assumed that the joining of the pipes with a clamping ring to be axisymmetric behavior, due to their tubular shapes. Therefore, we determined and meshed a two-dimensional section of the solution domain for an axisymmetric plane for the pipes and the ring with 4-node quadratic elements. We used a total of 5,000 elements to get a space of $0.1 \mathrm{~mm}$ between nodes. At the contact lines between the pipes and between the outer pipe and the ring, we applied the Coulomb Friction model for the contact conditions, considering each contact to be only in a dry mechanical contact condition. We carried out the numerical analysis for the pipe connection with the finite element method using the commercial software ABAQUS[15]. In the numerical simulation for the joining process, we fed the clamping ring with a constant displacement of $1 \mathrm{~mm}$ onto the outer pipe in the axial direction for each static analysis step with changing the boundary condition at the front side of the ring. In this study, the analysis consisted of a total of 13 steps to simulate the whole connection process.

Table 1. Material properties of the pipe and clamping ring.

\begin{tabular}{|c|c|c|c|c|c|c|}
\hline Part & Material & $\begin{array}{c}\text { Yield } \\
\text { strength } \\
(\mathrm{Mpa})\end{array}$ & $\begin{array}{c}\text { Tensile } \\
\text { strength }(\mathrm{Mpa})\end{array}$ & Elongation & $\begin{array}{c}\text { Elastic } \\
\text { modulus }(\mathrm{Gpa})\end{array}$ & $\begin{array}{c}\text { Poisson's } \\
\text { ratio }\end{array}$ \\
\hline Pipe & Mild Steel & 200.0 & 400.0 & 0.3 & 210 & 0.3 \\
\hline Clampingring & Hardened Steel & 638.5 & 704.8 & 0.06 & 210 & 0.3 \\
\hline
\end{tabular}

\section{B. Contact forces for design parameters}

In order to estimate contact forces between the two pipes in the mechanical joint with a clamping ring for the various radii and thicknesses of the pipes and the rings used in the related industries, Table 2 shows that we selected a total of 27 cases of joints to analyze to obtain radial stress distributions in the solution domain with the proposed numerical model. Each ring for the analyses had a length of $9 \mathrm{~mm}$ and a taper of $8^{\circ}$ at the leading section. In addition, Fig. 2 shows that we calculated the radius of contact position of $r$ over the contact length of L for each joint as shown in Fig. 2 through mechanical deformation analyses.

We then derived the contact force, $\mathrm{N}$, for each joint from integration of the radial stresses, $S_{r r}$, over the contact area between the two pipes, as follows:

$$
\mathrm{N}=\int 2 \pi r S_{r r} \mathrm{dL}
$$


Table 2. Twenty-seven sets of design parameters for analysis of the contact force of the pipe connection with variation of the parameters.(unit: $\mathrm{mm}$ )

\begin{tabular}{|c|c|c|c|c|c|c|}
\hline \multirow{2}{*}{ Set } & \multicolumn{2}{|c|}{ Inner pipe } & \multicolumn{2}{|c|}{ Outer pipe } & \multicolumn{2}{|c|}{ Clamping ring } \\
\hline & $r_{i}$ & $r_{0}$ & $\mathrm{r}_{\mathrm{i}}$ & $\mathrm{r}_{\mathrm{o}}$ & $r_{i}$ & $r_{0}$ \\
\hline 1 & 1.308 & 1.818 & 1.893 & 2.405 & 2.219 & 4.564 \\
\hline 2 & 1.523 & 2.033 & 2.108 & 2.620 & 2.434 & 4.439 \\
\hline 3 & 1.128 & 1.818 & 1.893 & 2.405 & 2.219 & 4.224 \\
\hline 4 & 1.343 & 2.033 & 2.108 & 2.620 & 2.434 & 4.779 \\
\hline 5 & 1.308 & 1.818 & 1.893 & 2.530 & 2.344 & 4.349 \\
\hline 6 & 1.523 & 2.033 & 2.108 & 2.745 & 2.559 & 4.904 \\
\hline 7 & 1.128 & 1.818 & 1.893 & 2.530 & 2.344 & 4.689 \\
\hline 8 & 1.343 & 2.033 & 2.108 & 2.745 & 2.559 & 4.564 \\
\hline 9 & 1.308 & 1.818 & 1.893 & 2.405 & 2.036 & 4.041 \\
\hline 10 & 1.523 & 2.033 & 2.108 & 2.620 & 2.251 & 4.596 \\
\hline 11 & 1.128 & 1.818 & 1.893 & 2.405 & 2.036 & 4.381 \\
\hline 12 & 1.343 & 2.033 & 2.108 & 2.620 & 2.251 & 4.256 \\
\hline 13 & 1.308 & 1.818 & 1.893 & 2.530 & 2.161 & 4.506 \\
\hline 14 & 1.523 & 2.033 & 2.108 & 2.745 & 2.376 & 4.381 \\
\hline 15 & 1.128 & 1.818 & 1.893 & 2.530 & 2.161 & 4.166 \\
\hline 16 & 1.343 & 2.033 & 2.108 & 2.745 & 2.376 & 4.721 \\
\hline 17 & 1.110 & 1.710 & 1.785 & 2.360 & 2.083 & 4.258 \\
\hline 18 & 1.540 & 2.140 & 2.215 & 2.790 & 2.513 & 4.688 \\
\hline 19 & 1.145 & 1.925 & 2.000 & 2.575 & 2.298 & 4.473 \\
\hline 20 & 1.325 & 1.925 & 2.000 & 2.450 & 2.173 & 4.348 \\
\hline 21 & 1.325 & 1.925 & 2.000 & 2.700 & 2.423 & 4.598 \\
\hline 22 & 1.325 & 1.925 & 2.000 & 2.575 & 2.480 & 4.655 \\
\hline 23 & 1.325 & 1.925 & 2.000 & 2.575 & 2.115 & 4.290 \\
\hline 24 & 1.325 & 1.925 & 2.000 & 2.575 & 2.298 & 4.133 \\
\hline 25 & 1.325 & 1.925 & 2.000 & 2.575 & 2.298 & 4.813 \\
\hline 26 & 1.325 & 1.925 & 2.000 & 2.575 & 2.298 & 4.473 \\
\hline 27 & 1.505 & 1.925 & 2.000 & 2.575 & 2.298 & 4.473 \\
\hline
\end{tabular}

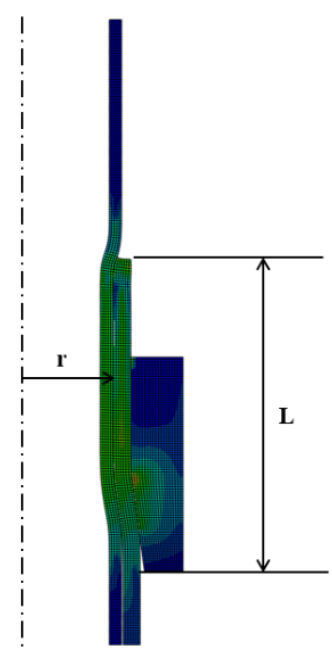

Fig. 2. Definition of the contact radius and contact length. 
After the series of analyses for the 27 cases of pipe joints, we utilized the contact force and corresponding design parameters for each joint at the same time to acquire their distinct relationship by using a nonlinear multi regression, which revealed the role and contribution of each parameter to the result of the contact force [12]. For the statistical approach, we presented the design parameters in a different way, as follows:

$\mathrm{x} 1$ : The outer radius of inner pipe, $\mathrm{r}_{\mathrm{o}}$, in the range between 1.71 and $2.14 \mathrm{~mm}$;

$\mathrm{x} 2$ : The thickness of inner pipe, $\mathrm{r}_{\mathrm{o}}-\mathrm{r}_{\mathrm{i}}$, in the range between 0.42 and $0.78 \mathrm{~mm}$;

$\mathrm{x} 3$ : The thickness of outer pipe, $\mathrm{r}_{\mathrm{o}}-\mathrm{r}_{\mathrm{i}}$, in the range between 0.45 and $0.75 \mathrm{~mm}$;

$\mathrm{x} 4$ : The contraction size, which subtracted the inner radius of the ring, $\mathrm{r}_{\mathrm{i}}$, from the outer radius of outer pipe, $\mathrm{r}_{0}$, in the range between 0.19 and $0.92 \mathrm{~mm}$;

$\mathrm{x} 5$ : The thickness of clamping ring, $\mathrm{r}_{\mathrm{o}}-\mathrm{r}_{\mathrm{i}}$, in the range between 1.84 and $2.52 \mathrm{~mm}$.

The statistical analysis for the results and the design parameters finally resulted in a simplified equation. We could use the equation to represent the relation between contact force and design parameters to effectively obtain the optimal design parameters for the pipe joint, including the optimal ring size.

\section{Tightening forces of the pipe joint}

The tightening force in the axial direction of the pipe joint with a clamping ring can be estimated by the contact force and the friction coefficient between the two pipes, i.e., $F=\mu \mathrm{N}$. We believe the force to be the ability of the joint to resist being separated in the axial direction. That is to say, the joint can withstand an applied load up to the tightening force before the fracture of the joint. However, because the friction coefficient was not available for the contact condition, we could not estimate the tightening force from the contact force.

In this study therefore, we obtained the friction coefficient by matching the estimated tightening force of the analyses by changing the coefficient step-by-step with the tensile force we obtained before the fracture of the joint in the experiment. We prepared a group of pipe joints for the experiment and the analyses of the tensile test. Table 3 shows the sizes of the pipes and the ring used in the comparison. In particular, we prepared 5 specimens for the experiment, then processed with no application of anaerobic sealant.

Table 3.Design parameters for tensile tests of the pipe connection (unit: $\mathrm{mm}$ ).

\begin{tabular}{|c|c|c|c|c|c|}
\hline \multicolumn{2}{|c|}{ Inner pipe } & \multicolumn{2}{c|}{ Outer pipe } & \multicolumn{2}{c|}{ Clamping ring } \\
\hline $\mathrm{r}_{\mathrm{i}}$ & $\mathrm{r}_{\mathrm{o}}$ & $\mathrm{r}_{\mathrm{i}}$ & $\mathrm{r}_{\mathrm{o}}$ & $\mathrm{r}_{\mathrm{i}}$ & $\mathrm{r}_{\mathrm{o}}$ \\
\hline 1.60 & 2.10 & 2.15 & 2.85 & 2.485 & 5.005 \\
\hline
\end{tabular}

Figure 3 shows the experimental setup of the tensile test for the pipe joint in this study, which was performed with a pulling speed of $150 \mathrm{~mm} / \mathrm{min}$ to obtain the ultimate tensile load up to the fracture of the joint.

We then chose the friction coefficient for each group for the numerical model by matching the predicted tightening forces from changing the coefficient, with the mean value of the five experimental results.

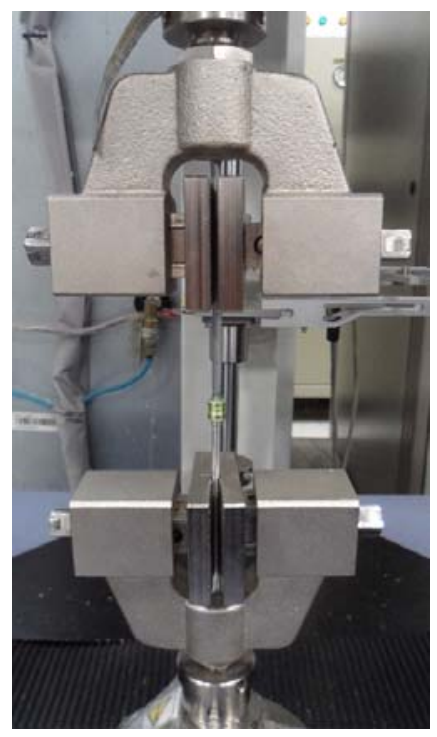

Fig. 3. Experimental setup of tensile test for pipe joint. 


\section{III.RESULTS AND DISCUSSION}

We modeled and analyzed the mechanical pipe joining process with a clamping ring by the numerical method of FEM. As the hydraulic machine pushed the ring onto the surface of the end of the outer pipe to acquire a mechanical tightening with the inner pipe, we analyzed the mechanical behaviors of the pipes and the clamping ring with the model to produce the deformed shape and internal stress distributions at each feeding step during the insertion of the ring we show in Fig. 4. Because the results show the deformed shape and equivalent stress distributions of the pipe joint with the clamping ring at each feeding step of the total of 13 steps, we could closely examine the change of deformed shape and stress distributions of the joint during the process. In particular, the final result shows the indentation left on the pipes corresponding to the inside profile of the clamping ring.

Figure 5 shows the radial stress distributions along the axial direction between the inner pipe and outer pipe of the connection joined for the 27th set of design parameters shown in Table 2 after the numerical analysis for the joining process. This produced a distribution of compressive stresses with two peaks at positions A and B, revealing a maximum stress of $-202 \mathrm{Mpa}$ at contact position A.

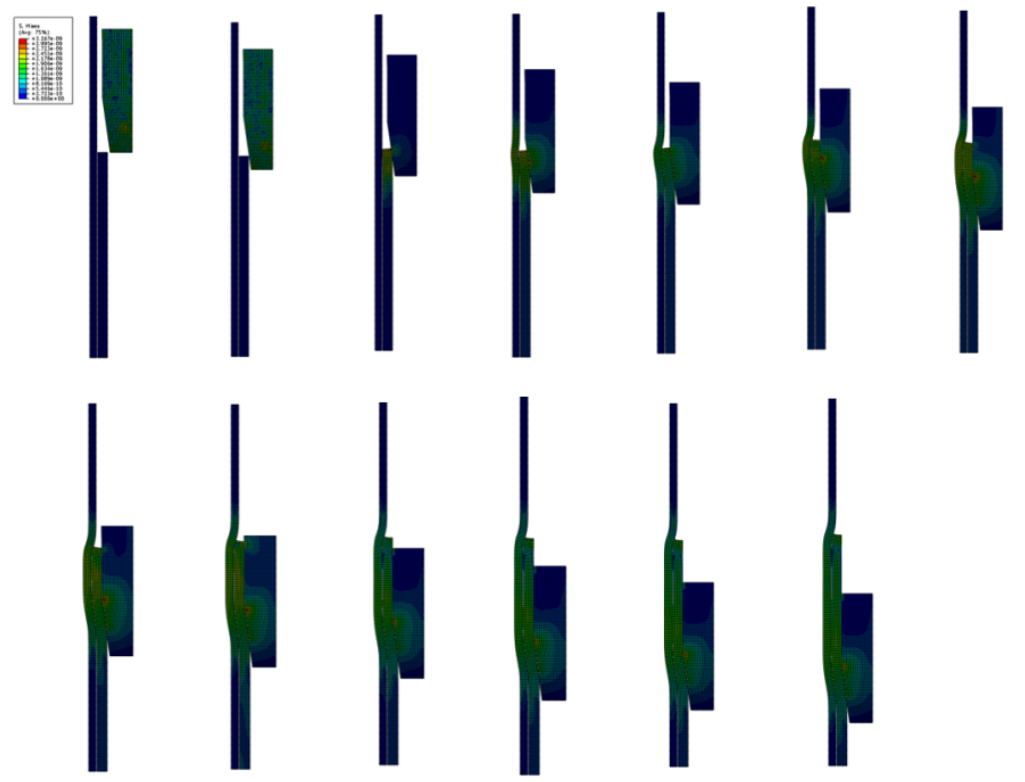

Fig. 4. Deformed shape and stress distributions of the pipe connection at each simulation step during the insertion of the clamping ring.
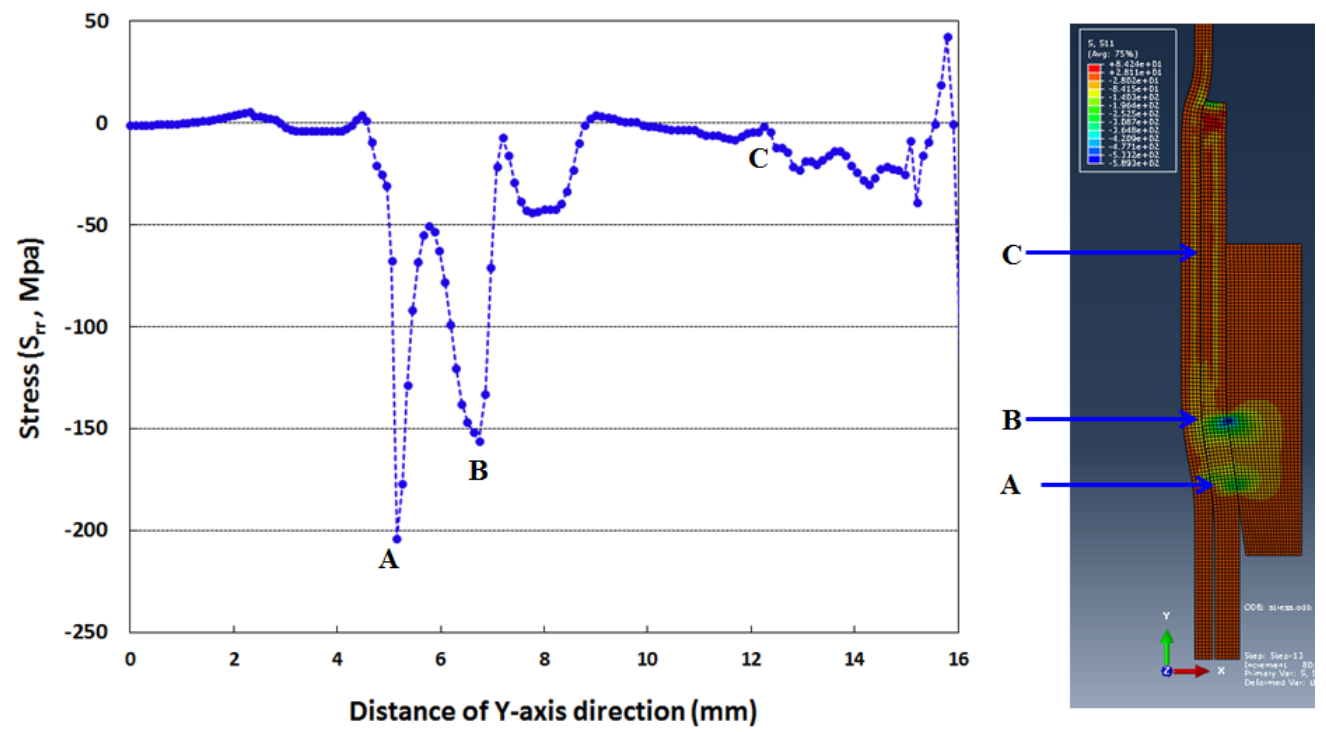

Fig. 5. Radial stress distributions between pipes along the axial direction of the pipe joint.

Figure 6 shows the equivalent stress distribution we obtained along the axial direction between the pipes joined with the 27th set of the parameters shown in Table 2. We can see from the result that those contact stresses were sufficiently high to plastically yield the pipe surface under the clamping ring, forming a metal-tometal seal between the pipes. The result also shows that plastic deformation was produced at the area from about 
$4 \mathrm{~mm}$ in the y-axis direction, where the equivalent stresses were greater than the yield strength of the pipe of 200 Mpa. Furthermore, the maximum stress presented at the point D nearly reached the tensile strength of the pipe of $400 \mathrm{Mpa}$. Therefore, we believe abrupt plastic deformations of the pipes occurred during the insertion of the clamping ring, as Fig. 4 shows.

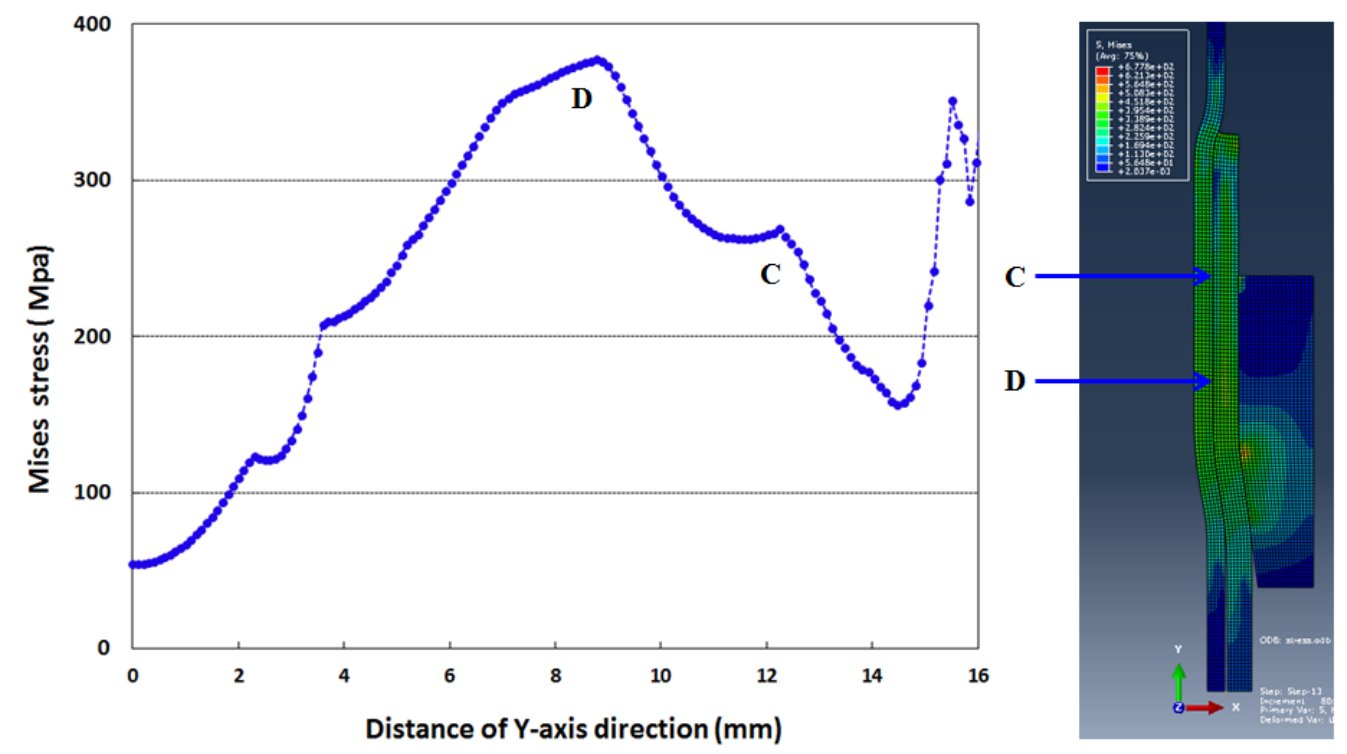

Fig. 6. Equivalent stress distributions between pipes along the axial direction of the pipe joint.

Figure 7 presents the deformed shape and contours of the equivalent stress distributions resulting from the analysis for the connection joined with the 27th set of parameters. The results show the deformed shapes of the pipes around the joining areas, and the actual contact sizes between the ring and the outer pipe. The enlarged contact of the joint around the location where the end part of the ring is positioned reveals that the contact between the clamping ring and the outer pipe was well established due to the inner profile of the ring.

Table 4 shows the maximum and average magnitude of the radial stresses along the contact area between two joined pipes produced by our numerical analysis for the 27 sets of design parameters shown in Table 2. The results show that the maximum stress varied from $142 \mathrm{Mpa}$ to $465 \mathrm{Mpa}$ according to the design parameters, whereas the average stresses changed from 20 to $55 \mathrm{Mpa}$ for the differing sets of design parameters.
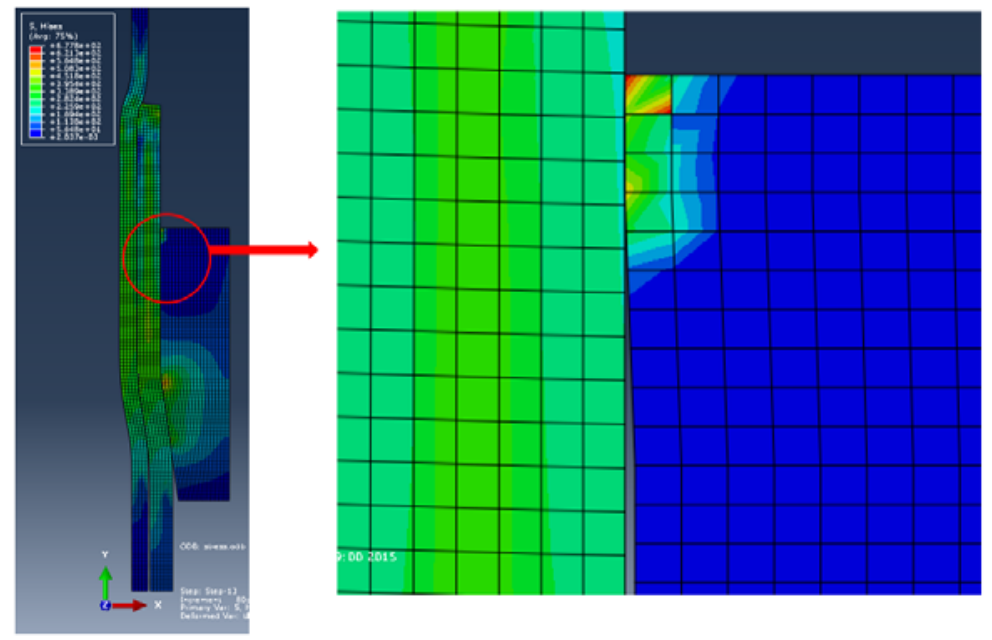

Fig. 7. Deformed shape and stress distributions predicted for the joint with a clamping ring, and enlarged contact area between the outer pipe and the ring.

Table 4 also presents the contact forces for different design parameters. We obtained the contact force by integration of the radial stresses over the contact area. In particular, Fig. 8 graphically illustrates the changes of contact forces with the increment of the contraction or thickness of the ring. The results show that the amount of contraction significantly influenced the contact forces, whereas the thickness of the ring did not much affect the force. Meanwhile, the simplified equation between the contact force and design parameters derived by the 
statistical analysis of non-linear multi-regression for the obtained results and the 27 sets of design parameters shown in Table 2 was as follows:

$$
\mathrm{N}=\frac{(42567)(x 2)^{1.408}(x 3)^{0.606}(x 4)^{0.144}}{(x 1)^{0.713}(x 5)^{0.063}}
$$

In the equation, we can clearly recognize the role played by and contribution of each design parameter to the contact force. In addition, the equation facilitates estimation of the change of contact forces of a joint for various set of design parameters for searching an optimal set of design parameters.

Table 4. Maximum and average radial stresses and contact force predicted for each set of design parameters in the numerical analyses for a total of 27 sets of design parameters.

\begin{tabular}{|c|c|c|c|}
\hline Set & Maximum (Mpa) & Average (Mpa) & Contact force $(N)$ \\
\hline 1 & 391 & 25.4 & 3512 \\
\hline 2 & 361 & 23.4 & 3639 \\
\hline 3 & 428 & 42.6 & 6135 \\
\hline 4 & 349 & 39.1 & 6285 \\
\hline 5 & 464 & 30.7 & 4285 \\
\hline 6 & 455 & 26.3 & 4109 \\
\hline 7 & 401 & 50.5 & 7106 \\
\hline 8 & 465 & 42.5 & 6892 \\
\hline 9 & 302 & 36.9 & 4607 \\
\hline 10 & 283 & 27.5 & 3878 \\
\hline 11 & 395 & 49.0 & 6266 \\
\hline 12 & 392 & 38.9 & 5599 \\
\hline 13 & 349 & 41.9 & 5253 \\
\hline 14 & 341 & 29.8 & 4226 \\
\hline 15 & 449 & 55.4 & 7123 \\
\hline 16 & 435 & 44.6 & 6449 \\
\hline 17 & 295 & 37.2 & 4615 \\
\hline 18 & 251 & 24.3 & 3802 \\
\hline 19 & 367 & 43.2 & 6169 \\
\hline 20 & 228 & 24.4 & 3410 \\
\hline 21 & 341 & 32.8 & 4613 \\
\hline 22 & 142 & 24.2 & 3621 \\
\hline 23 & 330 & 52.6 & 6777 \\
\hline 24 & 289 & 32.2 & 4509 \\
\hline 25 & 278 & 28.6 & 4005 \\
\hline 26 & 279 & 29.0 & 4061 \\
\hline 27 & 202 & 20.4 & 2808 \\
\hline
\end{tabular}




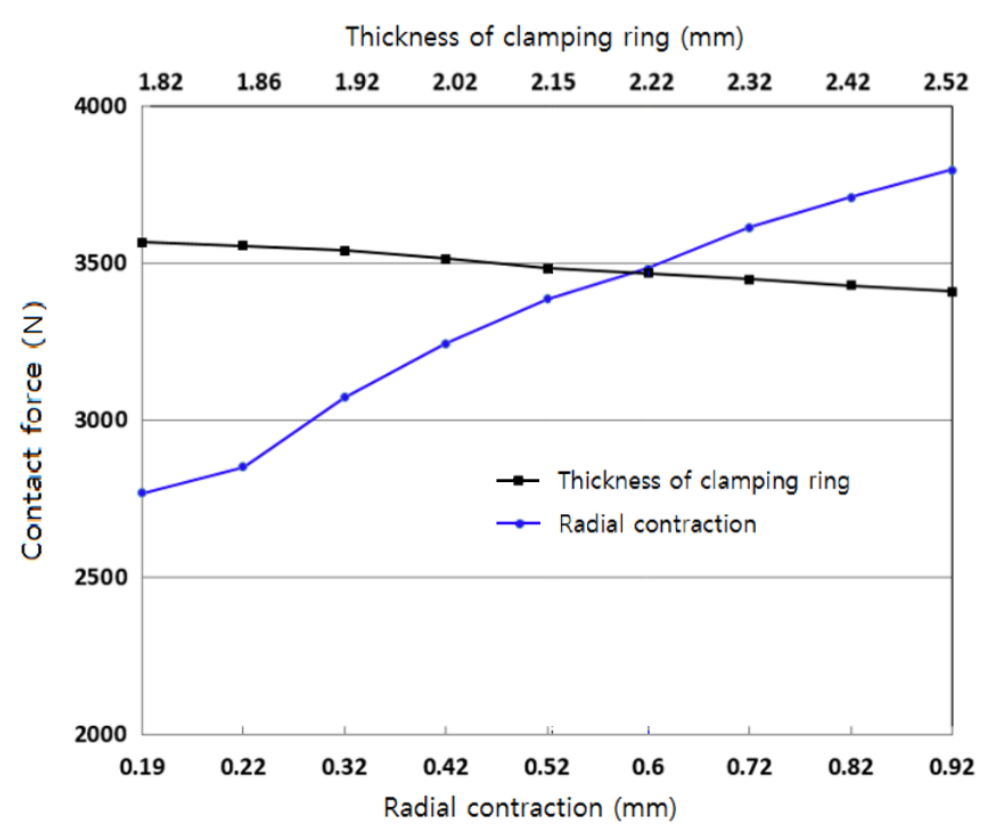

Fig. 8. Effects of radial contraction and thickness of clamping ring on the contact force.

Figure 9 compares the results of the contact forces obtained from the numerical analyses to those obtained by the simplified equation for all sets of design parameters, and shows them to be fairly well matched. Therefore, we could use the simplified equation to design the pipe joints by easily and effectively selecting the optimal sizes of the clamping ring to acquire the maximum contact force for the joints.

We experimentally measured the tightening forces of the pipe connection with a clamping ring for the design parameters shown in Table 3, with tensile tests performed up to the fracture of the joint. Table 5 shows the experimental results of the tensile tests for the 5 specimens of pipe joint, and it gives a mean value of $1452 \mathrm{~N}$. Because the deviation of the results was believed to be small enough, the tightening force of the joint could be presented as the mean value.

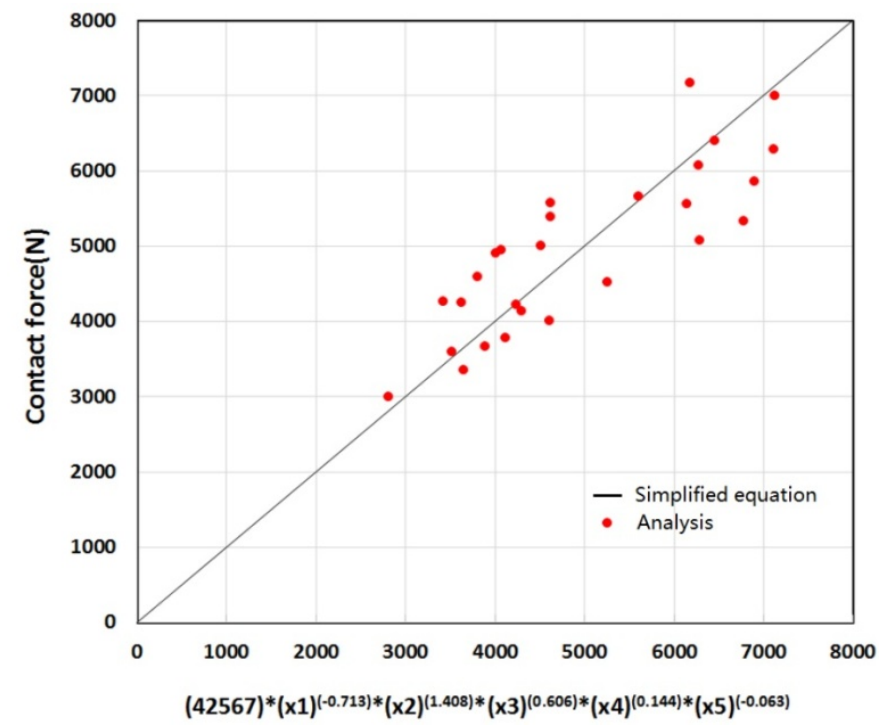

Fig. 9. Comparison of the predicted contact forces by the simplified equation with the results of analyses.

Table 5. Experimental results of tensile tests for 5 pipe joints.

\begin{tabular}{|l|c|c|c|c|c|c|}
\hline Specimen no. & 1 & 2 & 3 & 4 & 5 & Average \\
\hline Tensile force (N) & 1457 & 1446 & 1469 & 1435 & 1453 & 1452 \\
\hline
\end{tabular}


To obtain the tightening force from the contact force resulted from the numerical analysis with the relation of $\mathrm{F}=\mu \mathrm{N}$, we derived the friction coefficient of the joint by trial and error. In the analysis of the tensile test, we pulled the inner pipe with $1452 \mathrm{~N}$ in the axial direction, which figure we obtained experimentally, and assumed the friction coefficient between the contact positions between the pipes. Figure 10 shows that we subsequently reduced the coefficient until separation of the joint occurred. For the joint prepared without the anaerobic sealant, the pipe separated when the coefficient reached 0.3. Therefore, we considered the joint to have a friction coefficient of 0.3 .

Because we estimate the tensile forces required for yielding and fracturing of the pipe, which has a yield strength of $200 \mathrm{Mpa}$, a tensile strength of $400 \mathrm{Mpa}$, and a sectional area of $6.123 \mathrm{~mm}^{2}$, to be $1250 \mathrm{~N}$ and $2500 \mathrm{~N}$, respectively, we expected the pipe joint without the preparation of the sealant to have a tightening force of up to $58 \%$ of the ultimate tensile force for the pipe itself.
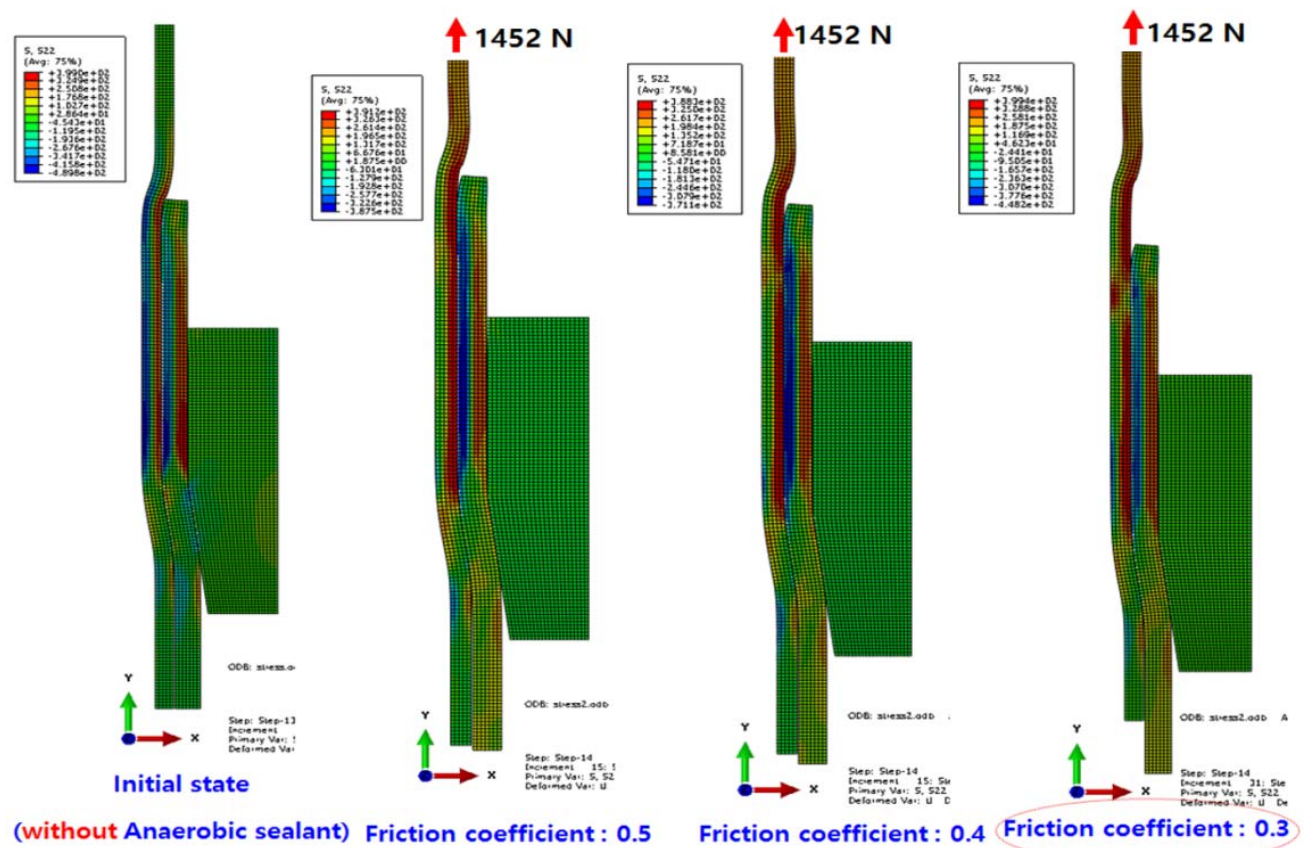

Fig. 10. Stress distribution of the deformed shape during the simulation of a tensile test with change of friction coefficient and with the load obtained as the maximum tensile force, in the experiment for the pipe joint without application of an anaerobic sealant.

\section{IV.CONCLUSION}

We proposed a numerical model to simulate the process of the pipe connection with a clamping ring, and then analyzed the mechanical behavior of the pipe joint by the finite element method. After a series of numerical simulations of the process for acquiring the effects of the sizes of the pipes and the rings on the contact forces between the pipes and then derivation of tightening forces of the joints, we obtained the following conclusions.

(1) The process of pipe connection with clamping ring could be modeled for numerical simulation to analyze contact and tightening forces of the joint.

(2) The contact forces between inner and outer pipes with variation of the pipe size and the ring size with respect to radius and thickness were produced, to analyze the effect of design parameters on the forces.

(3) The relation between the contact forces and the sizes of the pipes and the clamping rings was manipulated with the statistical technique of a recursion method, to obtain a simplified equation.

(4) The simplified equation could give optimized parameters for the sizes of the pipes and the clamping ring for a pipe connection with a clamping ring to increase the tightening forces.

(5)The tightening forces of the joint in the axial direction could be derived with the predicted contact forces between the pipes, by adjusting the friction coefficient through matching the forces with the result of the actual tensile test.

\section{ACKNOWLEDGMENT}

This study was financially supported by Samsung Electronics and Chonnam National University, 2015. 


\section{REFERENCES}

[1] B. Hackforth, "Clamping ring pipe connection for metallic pipes", Hackforth Gmbh \& Co. Kg, US patent 4911484 A, 1990.

[2] D. Schwalm, "Pipe connection having improved strength", VulKan Lokring R. Gmbh \& Co. KG, US patent 7654588 B2, 2010.

[3] "Refrigeration and air conditioning: solder-free tube connections for the installation and servicing", Vulkan Lokring Roh. GmbH \& Co. KG, Herne, Germany, 2009.

[4] "LP-110 installation procedure: lokring brass fittings for medical gas and vacuum applications", Lokring Technology LLC, Ohio, USA, 2008.

[5] "Lokring ESP fitting applications guide", Lokring Technology LLC, Ohio, USA, 2014.

[6] M. Biersteker, C. Dietemann, S. Sareshwala, and R. W. Haupt, "Qualification of non-standard piping product form for ASME code for pressure piping, B31 applications", The American Society of Mechanical Engineers, Vol. 210-1, Codes and Standards and Applications for Design and Analysis of Pressure Vessel and Piping Components, pp. 127-134, 1991.

[7] S. Hatton, L. Rumsey, P. Biragoni, D. Roberts, "Development and qualification of end fittings for composite riser pipe", Offshore Technology Conference, Houston, Texas, USA, May 2013.

[8] P. Chen, S. Zhou and Y. Shi, "Structure design and analysis of a locking band type quick opening end closure using a new saddleshaped sealing ring for natural gas filters", ASME 2015 Pressure Vessels and Piping Conference, Vol. 3, Boston, Massachusetts, UAS, July 2015.

[9] M. W. Kim, K. H. Lee, K. D. Hur and S. D. Ye, "Study on notch distance according to shape pipe fitting part", Conference Proceedings of KSME 2015 Annual Meeting, Jeju, pp. 2878-2882, Nov. 2015.

[10] K. H. Lee, S. D. Ye, M. W. Kim and K. D. Hur, "Pipe fitting molding process design", Conference Proceedings of KSPE 2015 annual meeting, pp. 357-358, Dec. 2015.

[11] W. Kim and C. M. Lee, "A study on the manufacture process parameters of stainless/carbon composite material pipes fitting", Conference Proceedings of KSMPE 2008 annual meeting, pp. 144-149, Nov. 2008.

[12] Norusis, M., SPSS 15.0, guide to data analysis, 2006, Prentice Hall, New York.

[13] "Technical documentation and submittal: aluminum connector type 50", Vulkan Lokring Roh. GmbH \& Co. KG, Herne, Germany, 2014.

[14] Metals Handbook, "Properties and selection: irons, steels, and high-performance alloys", 10th ed., Vol. 1, ASM, pp. 232-236, 1990.

[15] ABAQUS User's Manual, Version 6.7-2, Hibbitt, Karlsson and Sorensen Inc., Pawtucket, Rhode Island, 2007. 